FERMILAB-TM-1928

\title{
Coating Power RF Components with TiN
}

\author{
M. Kuchnir and E. Hahn \\ Fermi National Accelerator Laboratory \\ P.O. Box 500, Batavia, Illinois 60510
}

March 1995 


\section{Disclaimer}

This report was prepared as an account of work sponsored by an agency of the United States Government. Neither the United States Government nor any agency thereof, nor any of their employees, makes any warranty, express or implied, or assumes any legal liability or responsibility for the accuracy, completeness, or usefulness of any information, apparatus, product, or process disclosed, or represents that its use would not infringe privately owned rights. Reference herein to any specific commercial product, process, or service by trade name, trademark, manufacturer, or otherwise, does not necessarily constitute or imply its endorsement, recommendation, or favoring by the United States Government or any agency thereof. The views and opinions of authors expressed herein do not necessarily state or reflect those of the United States Government or any agency thereof. 
Fermilab TM-1928

\title{
Coating Power RF Components with TiN
}

\author{
M. Kuchnir and E. Hahn \\ Fermi National Accelerator Laboratory \\ Batavia, IL 60510 U S A \\ March 21, 1995
}

\begin{abstract}
A facility for coating RF power components with thin films of Ti and/or TiN has been in operation for some time at Fermilab supporting the Accelerator Division RF development work and the TESLA program. It has been experimentally verified that such coatings improve the performance of these components as far as withstanding higher electric fields. This is attributed to a reduction in the secondary electron emission coefficient of the surfaces when coated with a thin film containing titanium. The purpose of this Technical Memorandum is to describe the facility and the procedure used.
\end{abstract}

\section{INTRODUCTION}

After reading some of the literature $1,2,3,4,5$ we set ourselves to develop a capability for coating ceramic windows and metallic components with titanium nitride (TiN). The rational in selecting TiN is the expectation that components coated with it will require less processing time than components coated with pure titanium (Ti). TiN is very stable while pure Ti oxidizes when exposed to air and later when in vacuum under intense RF fields this oxide decomposes back leaving pure Ti on the surface therefore requiring some additional processing time. The contracting of a commercial sputtering laboratory proved to be too expensive for the few samples of many different shapes with which we will be working. Using the Lab 7 facilities of the Physics Department we have developed this procedure for coating by sublimating the $\mathrm{Ti}$ from an electrically heated $\mathrm{Ti}$ filament in an ammonia $\left(\mathrm{NH}_{3}\right)$ atmosphere onto the sample surface. This way of proceeding has been suggested by work ${ }^{6}$ from LANL. 


\section{APPARATUS}

The system we use consists of a cylindrical stainless bell-jar, of about $0.5 \mathrm{~m}$ diameter and about $1 \mathrm{~m}$ tall sitting over a base with several KF flanges (for feedthroughs and accessories) and a pumping system based on a turbo-pump. Overnight we usually achieve a base vacuum better than $1 \times 10^{-5}$ torr. The bell-jar itself has several large KF flanges, two of which we use in this application: one with a quartz window for an optical pyrometer and the other with an ultraviolet lamp for water desorption. Among the instrumentation in this system we have several vacuum gauges (a convectron ${ }^{7}$, a Penning pressure gauge ${ }^{8}$ and a Bayard-Alpert ion gauge ${ }^{9}$ ), a film thickness monitor ${ }^{10}$ which is based on the resonant frequency of replaceable quartz crystals and a thermocouple. The last two as well as the external pyrometer ${ }^{11}$ are movable so that they can be positioned for properly monitoring the sublimation operation. The pyrometer is based on the ratio of the intensities of two wavelengths and requires no accurate surface emissivity knowledge. It sees the filament through a $12.7 \mathrm{~mm}$ thick fused quartz window ${ }^{12}$ that has a 0.94 transmission factor for the $0.7 \mu \mathrm{m}$ and $1.08 \mu \mathrm{m}$ wavelengths.

Two gas handling manifolds are used with this system: One connected via regulator to a cylinder with $\mathrm{N}_{2}$ gas $99.999 \%$ pure for purging and venting the system and the other connected via metering valve ${ }^{13}$ and regulator to a lecture bottle ${ }^{14}$ with $\mathrm{NH}_{3}$ gas $99.99+\%$ pure for the chemical vapor deposition operation. The safe use of $\mathrm{NH}_{3}$ involves extra vent piping for the vacuum pumping system and its regulator, insuring that all venting is done to the outside of the building.

The filaments are made out of titanium welding rods, $1.5 \mathrm{~mm}$ diameter and are energized from a $1 \mathrm{~kW}$ DC power supply ${ }^{15}$ via $6.35 \mathrm{~mm}$ diameter copper rods and feedthroughs. The electrical connections are made by means of stainless screws radially positioned through the thick walls of $\mathrm{BeCu}$ sleeves. These sleeves come in 2 telescoping sizes that permit accommodating the difference in diameters.

For redundancy two data acquisition systems are associated with this apparatus: one consists of a 3 channel chart recorder/plotter ${ }^{16}$ and the other is based on a 10 channel digital multimeter ${ }^{17}$ and a personal computer running a Basic program using IEEE-488 
for interface. At present there is no computer controlled operation in this system. Analysis of the data is done using Excel spreadsheet.

\section{SUBLIMATION SET UP.}

Each different power RF component or sample to be coated requires a custom arrangement of filaments geometry, shields and sublimations sequence. The geometry should be simple to permit calculations of the exposure parameters and estimates of deviations from uniformity. These calculations are also carried out for the location and orientation of the thickness monitor so that we have a guiding parameter during the exposure although the thickness monitor reading is affected by the radiation from the filament and its instantaneous reading requires corrections. Typical calculations are presented later on.

We have found that a new filament requires an extra operational step, a preliminary heating, for desorbing it of impurities, after which it looses its ductility, but remains quite usable.

We also found that when hot (at sublimating temperatures) the filaments loose all their rigidity and perhaps even elongate themselves just due to their own weight. Therefore they can be best used hanging as vertical lines or catenaries. The lack of rigidity in the filament when hot also requires that the leads be positioned to be free standing next to the connectors so that the connections once done apply no stress to the filament. Otherwise large unpredictable movements will occur when the filament gets hot ruining the set up.

Nevertheless, we have been successful in using filaments in the form of horizontal circles and even vertical circles by providing electrically isolated support points that withstand the temperatures and add little heat drain. This allow us to do without having the sample on a rotating platform. So with the help of Ti cold supports we use horizontal circular filaments $(13 \mathrm{~cm}$ and $28 \mathrm{~cm}$ diameters $)$ and vertical circular filaments $(2.5 \mathrm{~cm}$ diameter) when appropriate. The fixtures used to hold the filaments are made out of Aluminum, Copper, Stainless or Macor (a machinable ceramic) often using ceramic "stand-off" insulators. Parts that directly touch the filaments are made of Titanium, Macor or are the Beryllium Copper connectors at their ends.

The apparatus includes a thermocouple to monitor the temperature of the substrate since it affects the nature of the coating, but up to this point we have not made a systematic study of it. 
Besides the electrical feedthroughs for the filaments the apparatus also includes one for a wire that allows us to establish electric potential differences among the filaments, the walls of the vacuum chamber and the metallic parts of the components being coated. We had some evidence that keeping the filament positively charged $(9 \mathrm{~V})$ with respect to the substrate is important.

\section{COATING OPERATION}

All the parts internal to the vacuum chamber are kept clean and fingerprint free. The last cleaning operation usually being ultrasounding in very pure ethanol. Microwave windows, many times required their alumina $\left(\mathrm{Al}_{2} \mathrm{O}_{3}\right)$ ceramic to be sandblasted first, and this is done using $\mathrm{Al}_{2} \mathrm{O}_{3}$ powder.

Once the sublimation setup is completed (filaments connected, substrate component and thickness monitor positioned, substrate grounded or connected to electrical feedthrough, thermocouple attached) distances and angles between filament, substrate and thickness monitor are measured and Polaroid pictures of the setup are taken. This is also the time for measuring the resistances of the filaments and check for shorts.

The vacuum chamber is then closed and the vacuum purging operation started. The chamber is evacuated to a pressure of about 1 torr and refilled with ultra pure $\mathrm{N}_{2}$ gas three times. The resistance of one filament and the convectron signal are usually monitored in this operation, the changes in filament temperature (as detected in the resistance) is clearly noticeable. The system is then left pumping, with the turbo pump on, overnight, the Bayard-Alpert ion gauge being monitored. Typically a vacuum of the order of $1 . \times 10^{-6}$ torr is obtained. The filaments are connected to the power supply, the bias battery $(9 \mathrm{~V})$ connected, a thermometer set up to monitor the filament feedthrough that might get too hot, the back-up recorder is turned on, the thickness monitor zeroed and the recording program started.

Although there have been variations in this process a typical coating operation starts by setting a current of $21.5 \mathrm{~A}$ through the filament. This current is not sufficient to cause much sublimation but the filament becomes quite visible (dull orange) and there is plenty of time to setup and aim the pyrometer at the filament to monitor its temperature (about $1500 \mathrm{~K}$ ), to admit $\mathrm{NH}_{3}$ gas in the chamber and adjust its pressure to a stable value corresponding to a mean free path equal to the average distance between filament and substrate, all the while warming the substrate by infra red radiation. The coating proceeds by raising the current to about $27.5 \mathrm{~A}$ corresponding to a pyrometer reading of 
$2760^{\circ} \mathrm{F}(1789 \mathrm{~K})$ for the period determined by the exposure calculation, usually a few minutes.

If more than one filament is involved the current is turned off, and another filament connected to the power supply for another exposure. After the exposures the setup is then left to cool, the pumping stopped, more $\mathrm{NH}_{3}$ gas admitted to bring the pressure to a few torr and sometimes this condition is left for days until ready to get the coated component out. At which point the ammonia gas is pumped out, the vacuum chamber filled with $\mathrm{N}_{2}$ gas and then opened to the atmosphere. Examination of the color of the coating at this time is done, it is a qualitative and rather revealing test of the process from which improvements are devised.

In the development of the coating procedure described above, tests were carried out using alumina and glass sample substrates, on the sublimation of pure Ti (without $\mathrm{NH}_{3}$ gas) by measuring the resistivity of the coating during deposition. The admission of air dramatically increased the resistivity (Ti oxide is an insulator). Also the white color of titanium oxide makes it invisible on a white alumina substrate. So samples that after evaporation have a grayish color become white in a few hours of exposure to air. The gold color of TiN is much more stable. Many times the coating procedure is intentionally designed to result in a mixture of $\mathrm{Ti}$ and TiN. Then the resulting goldgrayish color on an alumina surface right after coating becomes with time just goldish. Although we aim for a specific coating thickness of $10 \mathrm{~nm}$, we expect that it is achieved only in order of magnitude. Nevertheless the performance of these coatings are rather effective in reducing multipactoring.

\section{EXPOSURE CALCULATIONS}

Lack of prompt feedback on the performance of the coatings and the relatively low duty cycle of the sublimation facility use has precluded the achievement of an optimal continuously improving operation. The low duty cycle of the sublimation facility causes its vacuum chamber to be modified for other uses and the associated equipment (power supply, data acquisition etc.) to be idle for months. In this regime drifts in calibrations, re-learning curve effects take their toll. The contents of this section was created during the conception of this coating by sublimation process and we hope that the inclusion of a multipactoring measuring system to this facility will lead to its refinement and optimal operation. 
The temperature of the filament varies along its length depending on whether it is touching supports or connectors. Here we refer to the temperature of the filament as its temperature in a point far away from these colder spots. So far this effect has been neglected or corrected by an approximation to the length of the filament.

The temperature dependence of the resistivity of $\mathrm{Ti}$, is shown in Figure 1 . It is interesting to observe in it the signature of a phase transition occurring at $1157 \mathrm{~K}$.

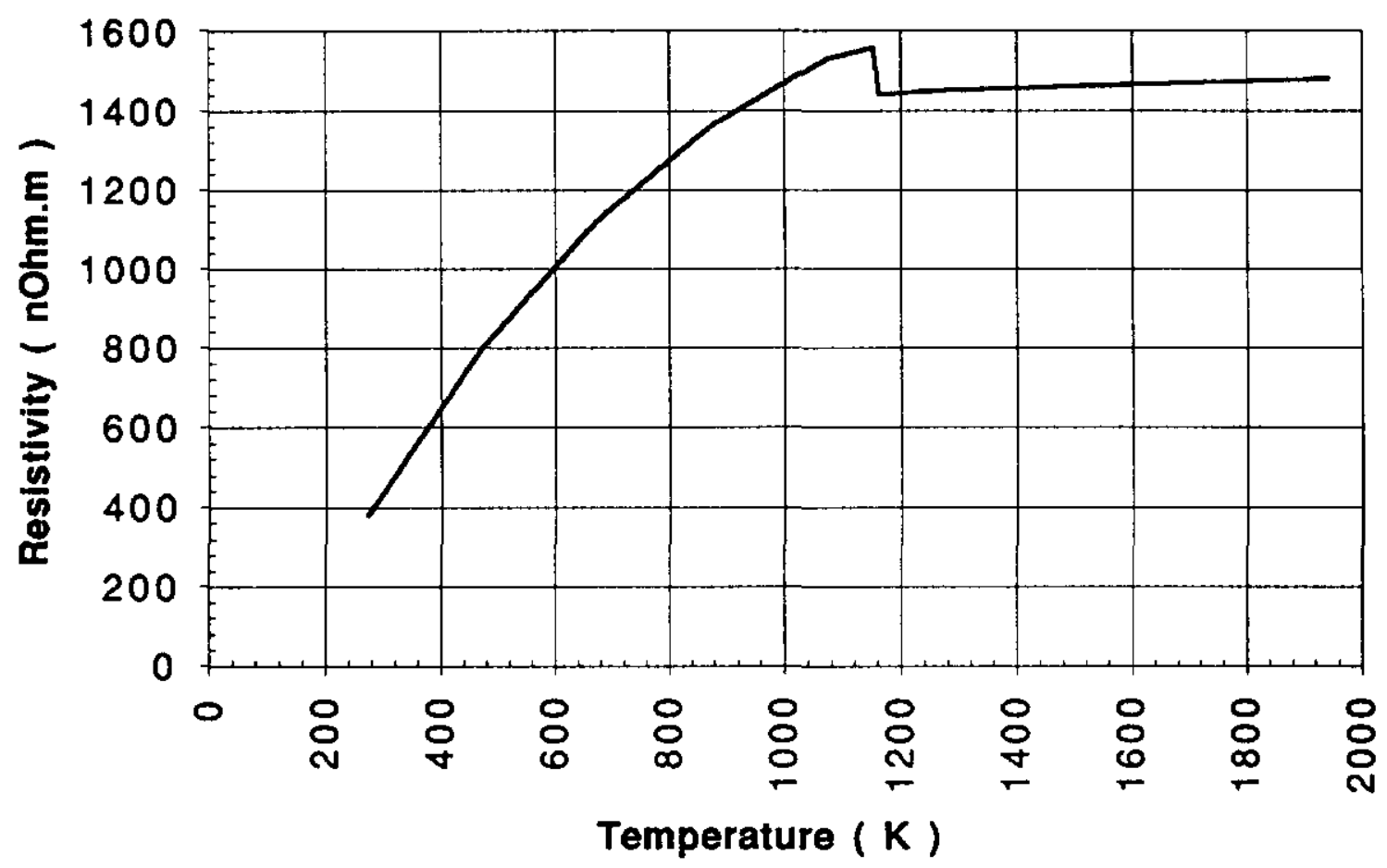

Figure 1. Titanium Resistivity

This temperature dependence in the region of interest is not sensitive enough for us to use the filament resistance as an indicator of its temperature ${ }^{18}$. We had, therefore, to invest in the optical pyrometer and the quartz window mentioned above with which we took the data shown in Figure 2.

The linear expression:

$$
\mathrm{T}=35.45 \cdot \mathbf{i}+814.5
$$

with $\mathrm{T}$ in Kelvins and $\mathbf{i}$ in Amperes represents this data for a filament of $1.52 \mathrm{~mm}$ diameter. The coefficients depend slightly and in an understandable way on the wear of the filament. Coating of the quartz window through which the pyrometer sees the 
filament and/or calibration drift of the pyrometer has caused changes in this expression with time. The pyrometer calibration can be and has been tested at $1941 \mathrm{~K}$, the melting point of Ti.

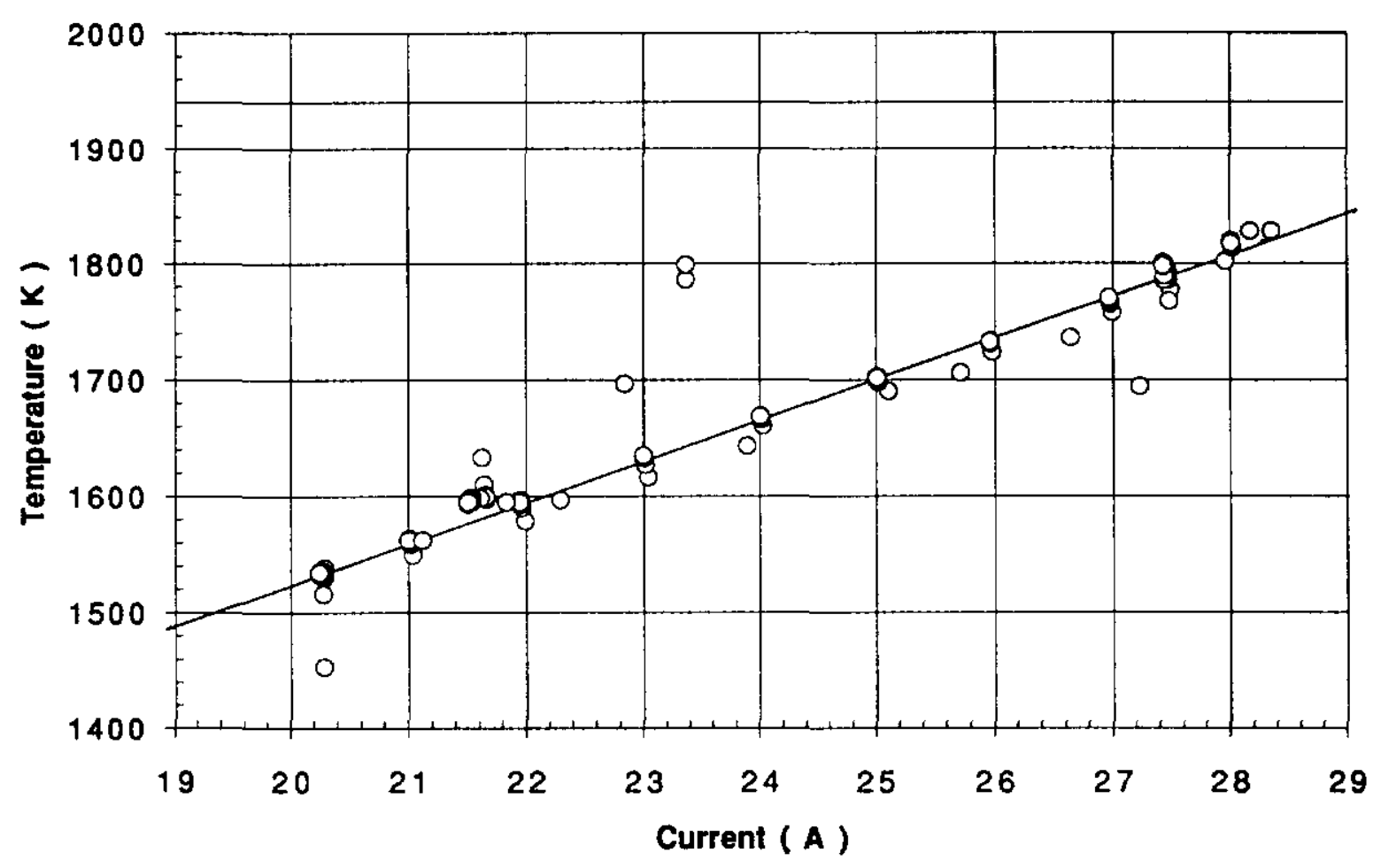

Figure 2. Filament temperature as function of the current

The vapor pressure curve of $\mathrm{Ti}$ among other metals ${ }^{19}$ in the region of interest can be represented by

$$
\log P=(-19.72+0.009202 \cdot T)
$$

where again $\mathrm{T}$ is in Kelvins and $\mathrm{P}$ is in torrs.

The following result from the kinetic theory of gases, called "Langmuir's Law",

$$
\mathbf{w}=0.585 \cdot \mathrm{P} \cdot(\mathrm{M} / \mathrm{T})^{1 / 2}
$$

where $\mathrm{M}$ is the molecular weight (47.9 for Ti), gives the mass of Ti sublimated per unit time and per unit area of filament surface. The coefficient here is for $T$ in Kelvins, $P$ in torr and $\mathrm{w}$ in $\mathrm{kg} \cdot \mathrm{m}^{-2} \cdot \mathrm{s}^{-1}$. With these expressions and the radiation inverse square law applied to the geometry of the filament plus sample surface system we can calculate exposure times for a given filament current and deposited Ti mass per unit surface of sample. 
The geometrical part of the calculation has been done in two ways, from first principles using calculus and algebra with the help of TK Solver Plus ${ }^{20}$, or using a computer code ${ }^{21}$ developed for the calculation of a 3-dimensional dose distribution generated by a radioactive line source of any length and shape. For this particular application the code was modified ${ }^{22}$ to give the photon fluence distribution in free space per unit source strength. Figure 3 are two typical outputs of this code one for a vertical filament and the other for a circular filament, these plots in actual size are to be compared with cross sections in actual dimensions of the samples in order to have an estimate of the deposition uniformity.

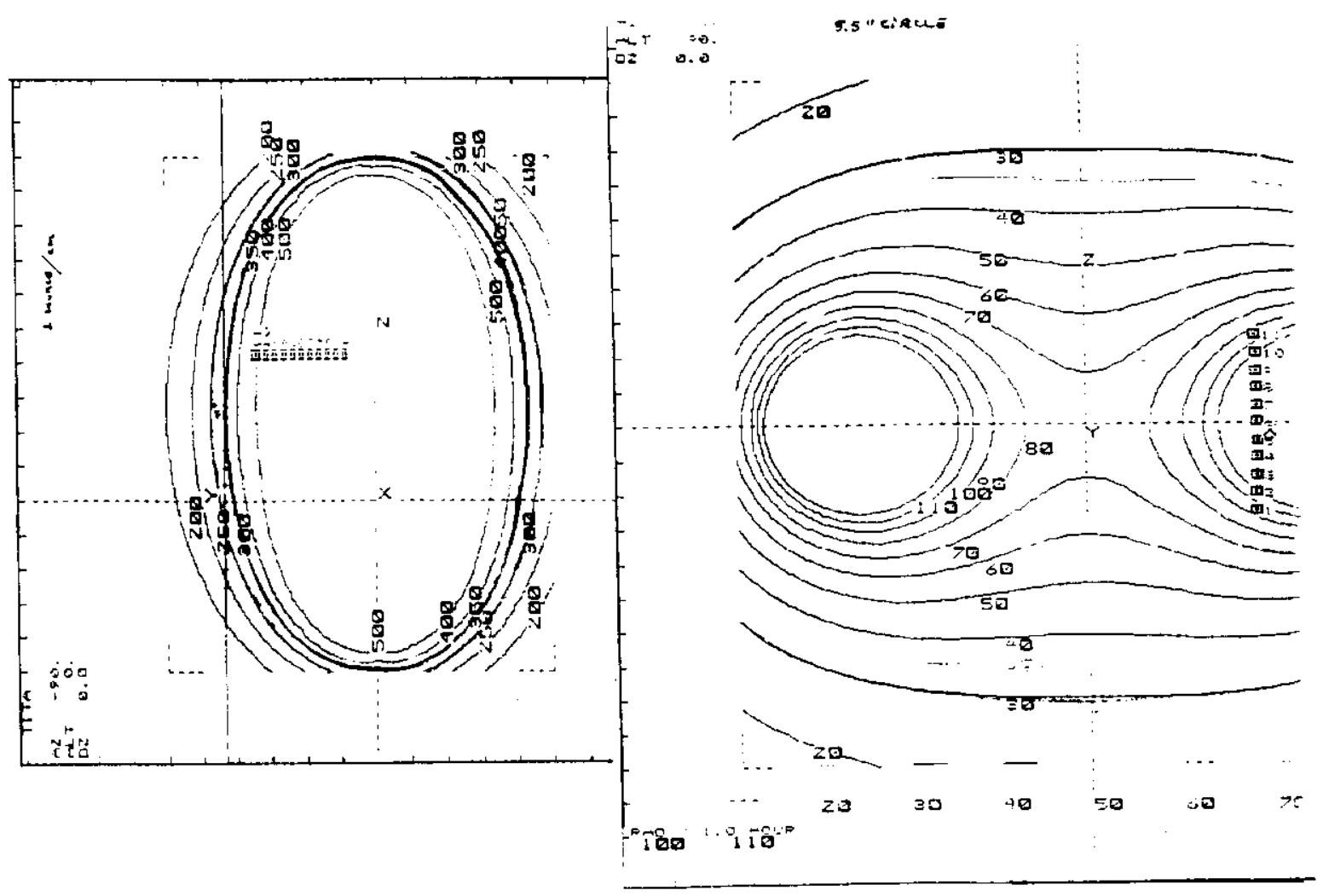

Figure 3. Isodoses from a vertical and a circular filaments

The exposure time that results from the calculations is a plot like the one in Figure 4 made for a deposited thickness of $10 \mathrm{~nm}$.

As described above the pressure of $\mathrm{NH}_{3}$ in the bell-jar during coating should correspond to a mean free path equal to the average distance between the filament and the surface of the sample. This pressure was calculated from the expression 23

$$
\mathrm{L}=2.33 \times 10^{-20} \cdot \mathrm{T} /\left(\mathrm{P} \cdot\left(4.42 \times 10^{-8}\right)^{2}\right)
$$

and is usually in the $1 \times 10^{-4}$ torr range. A rather useful plot of it is shown in Figure 5 . 


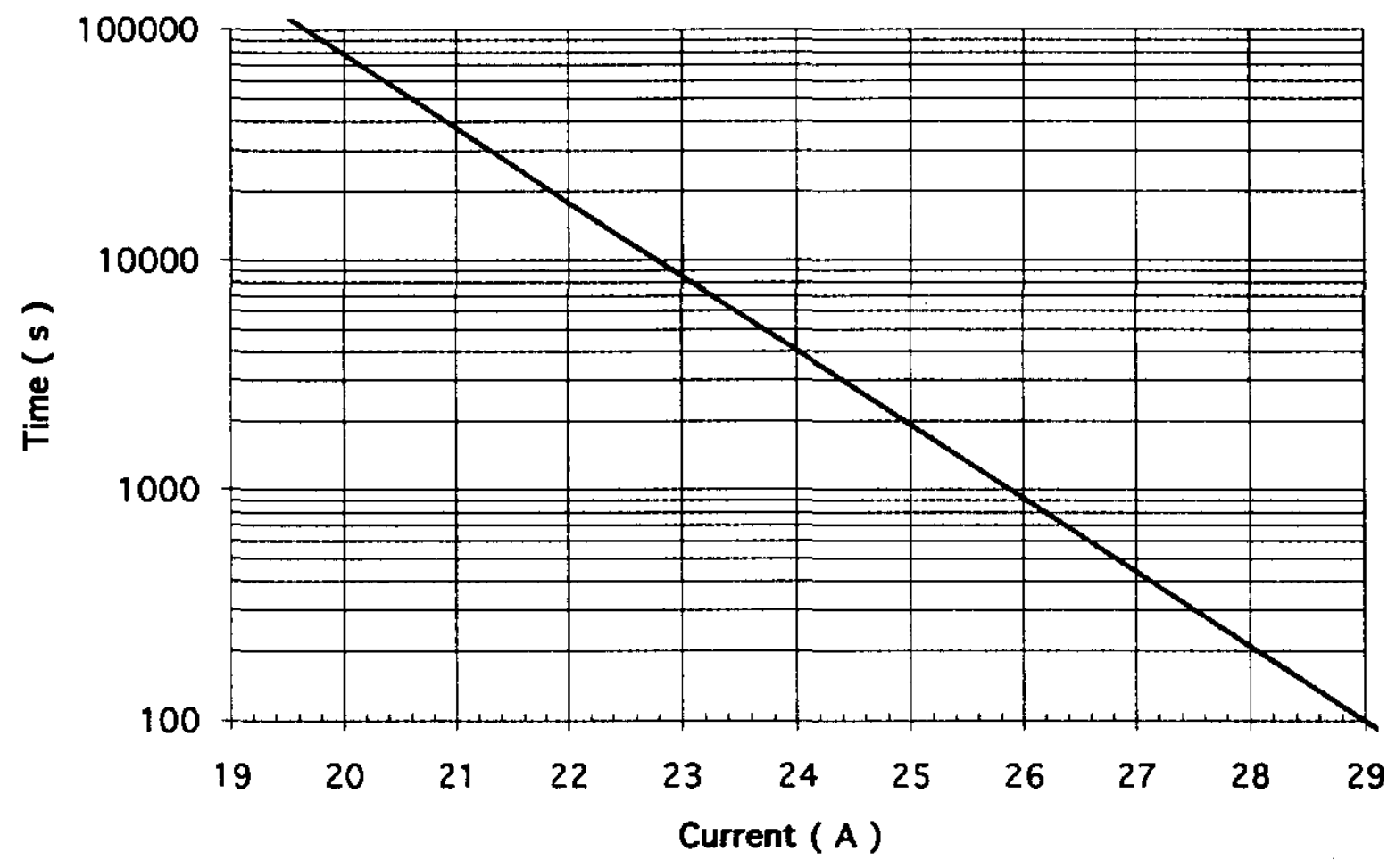

Figure 4. Typical exposure plot for guiding a sublimation run.

\section{COMMENTS}

We cannot use the film thickness monitor very effectively to monitor an exposure in real time because the heat and the electron flux radiating from the filament affect its reading. However, its indication after an exposure is usually in reasonable agreement with the expected coating thickness when properly corrected for its geometric arrangement with respect to the filament.

Early in the development of this operation we used the procedure of monitoring the exposure with the electrical resistivity of an alumina slide strategically exposed but this method works only when the sublimation is done without $\mathrm{NH}_{3}$ or $\mathrm{N}_{2}$. The resistivity of the slide dramatically changes with time upon its exposure to these gases or air. The admission of $\mathrm{NH}_{3}$ after the sublimation was not very effective in producing the gold colored signature of a TiN coating. Also $\mathrm{N}_{2}$ gas was not as effective as $\mathrm{NH}_{3}$ for this purpose. Although the apparatus can be fitted with a liquid $\mathrm{N}_{2}$ trap and using it the base pressure gets reduced by a factor of 2 to 5 , this trap cannot be used with a $\mathrm{NH}_{3}$ atmosphere. A desorption ultraviolet lamp was used in the early days to improve the 
pump down time but we noticed that overnight exposure to it discolored the alumina ceramic making it uniformly yellowish.

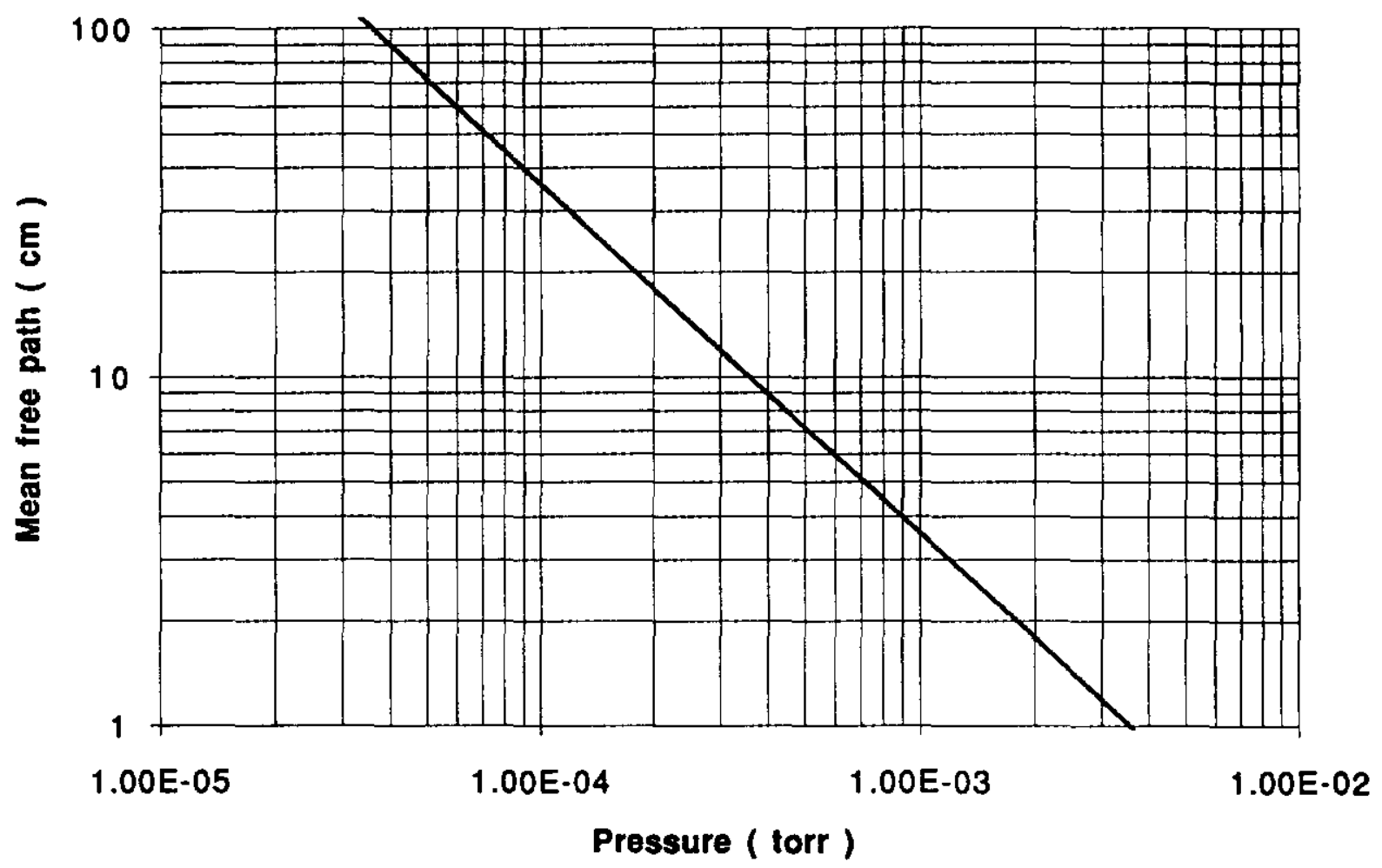

Figure 5. Mean free path vs. Pressure for $\mathrm{NH}_{3}$.

\section{RESULTS}

We have been successful in producing coatings that significantly reduce multipactoring in the treated power RF components as verified by the change in performance before and after coating of a proposed Tevatron beam position detector, a TESLA doorknob, a PETRA II cavity window ${ }^{24}$, and as inferred from many other components that were coated from the start and performed adequately. Nevertheless, there are several aspects of our operation that could be improved.

The most unsatisfactory aspect of the operation is the long feedback time between the coating operation and the report of the component performance under high power. We have given some thought about installing an electron gun and a Faraday cup in our system so that we can evaluate the performance of the coatings on the spot, but that has not been implemented yet. 


\section{REFERENCES}

${ }^{1}$ A.R. Nyaiesh, E.L. Garwin, F.K. King and R.E. Kirby, "Properties of thin antimultipactor $\mathrm{TiN}^{2}$ and $\mathrm{Cr}_{2} \mathrm{O}_{3}$ coatings for klystron windows", J. Vac. Sci. Technol., vol. A4(5), pp. 2356-2363, (1986).

${ }^{2}$ Frank Krienen, "On the Coating of SLAC Klystrons Windows", SLAC/AP-23, (May 23, 1984).

${ }^{3}$ A.R. Nyaiesh, E.L. Garwin, F.K. King and R.E. Kirby, "Properties of Thin Anti-Multipactor Coatings for Klystron Windows", SLAC-PUB-3760, (August 1985).

${ }^{4}$ Peter J. Clarke, "Magnetron dc reactive sputtering of titanium nitride and indium-tin oxide", J. Vac. Sci. Technol., vol. 14(1), pp. 141-142, (1977).

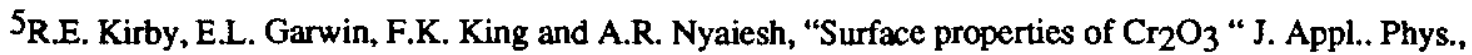
vol. 62(4), pp. 1400-1405, (1987).

${ }^{6}$ Wayne D. Cornelius and Robert J. Grieggs, "Elimination of Multipactoring in the FMIT RFQ Accelerator", Publication LA-UR-84-2489, Los Alamos National Laboratory.

${ }^{7}$ Model 275 Convectron Gauge/Digital Controller, manufactured by Granville-Phillips Company, 5675 Arapahoe Ave., Boulder, CO 80303-1398, U.S.A. (800)776-6543

8Penning Cold Cathode Gauge Type GPH-001A sensing tube used with Type GPH-320C gauge circuit, manufactured by CVC Products, 525 Lee Rd. Rochester, NY 14606, U.S.A. (800)448-5900

${ }^{9}$ Model 340 Bayard-Alpert Ion Gauge/Controller, manufactured by Granville Phillips Company, 5675 Arapahoe Ave. Boulder, CO 80303-1398, U.S.A. (800)776-6543

10Model STM-100/MF Thickness/Rate Monitor, manufactured by Sycon Instruments Inc., 6757 Kinne St. East Syracuse, NY 13057-1215 U.S.A. (315)463-5297

11 Model \#MR-OR 10-40F-0-0/0-1-0.MIRAGE two-color/ratio pyrometer manufactured by IRCON Inc., 7301 N. Caldwell Ave., Niles, IL 60714, U.S.A.(800)323-7660

12Fused Quartz window, obtained from Karl Lambrecht Corp., 4204 Lincoln Ave., Chicago, IL 60618 U.S.A. (312)472-5442

${ }^{13}$ Series 203 Variable Leak Valve, manufactured by Granville-Phillips Company, 5675 Arapahoe Ave. Boulder, CO 80303-1398, U.S.A. (800)776-6543

${ }^{14}$ Catalog No. 29,499-3 Lecture bottle of Ammonia and Z14,851-2 stainless regulator, Aldrich Chemical Company, Inc. 1001 W. Saint Paul Ave. Milwaukee, WI 53233 U.S.A. (800)558-9160

15 Model HP6032A Power Supply manufactured by Hewlett Packard Co.

16Model HP7090A Measurement Ploting System, manufactured by Hewlett Packard Co.

${ }^{17}$ Model 2001 Multimeter with scanner board, manufactured by Keithley Instruments, Inc. 28775 Aurora Rd., Cleveland OH 44139-1891, U.S.A. (216)248-0400

${ }^{18}$ Metals Handbook vol., $9^{\text {th }}$ ed, p. 815, .American Society for Metals 
${ }^{19}$ Product and Vacuum Technology Reference Book, Leybold Vacuum Products, Inc, section 18, p. 94, (1992).

20 TK Solver Plus, an algebra solving software product of Universal Technical Systems, Inc 1220 Rock Street, Rockford, IL 61101, U.S.A. (800)435-7887

${ }^{21}$ Department of Radiation Oncology - University of Chicago Hospitals,

${ }^{22}$ C. Pelizzari- private communication

${ }^{23}$ Saul Dushman, “Scientific Foundations of Vacuum Techniques”, p. 30, John Wiley \& Sons, Inc. (1962).

24I. Gonin, L. Kravchuk, G. Romanov, S. Tarasov, A. Gamp, R. Kaiser, W. Molinnus, M. Kuchnir, “A Study of Multipactor Phenomena in the 52 MHz PETRA II Cavity at DESY ", Proceedings of the Fourth European Particle Accelerator Conference EPAC94, London, V. Suller and Ch. Petit-Jean-Genaz Editors, vol. 3, pp. 2197-2199, World Scientific, Singapore, (1994) 